\title{
Studies on Quality Attributes of Chicken Burger Prepared with Spinach, Basil and Radish
}

\author{
Aly, A.A. and Hasnaa A. Morsy \\ Home Economics Department, Faculty of Specific Education, Benha University, Egypt
}

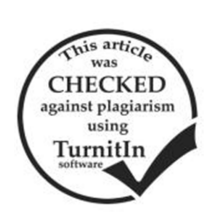

\section{ABSTRACT}

This study was carried out to utilize adding spinach, basil and radish to chicken burger formula and evaluation some chemical, sensory and bacteriological parameters. Radish (RB) and collected burger treatments had higher total lipids content (11.1\%). Crude protein content ranged between $49.1 \%$ and $64.2 \%$ in all chicken burger treatments. Ash, fiber and total carbohydrates content ranged between $7.6 \%$ and $10.5 \%, 1.86 \%$ and $3.25 \%, 17.04 \%$ and $31.65 \%$, respectively. The total saturated fatty acids for oils extracted from $\mathrm{CB}$ (Control burger), SB (spinach burger) and RB (radish burger) treatments were 29.65, 29.78and 33.96\%, while the total unsaturated fatty acids amounted to $68.04,69.97$ and $65.77 \%$, respectively and the palmtic acid was the major saturated fatty acids, while oleic acid was the predominant unsaturated fatty acid. Lycine was the predominant essential amino acid (4.66 \% to $5.36 \%)$ in all treatments. Glutamic acid showed higher ratio of non-essential amino acid (10.12\% and $12.19 \%)$. The results showed that addition of spinach, radish and basil to chicken burger samples retarded the growth of total molds and yeasts, total bacterial count, psychrophilic bacteria and spore-forming bacteria of chicken burger samples during freeze storage period $\left(-20 \pm 2{ }^{\circ} \mathrm{C}\right)$, hence the shelf life of radish and spinach burger samples increased to 8 months compared to other samples (6 months). Sensory properties such as appearance, color, texture, taste and odor of the chicken burgers samples were improved due to the applied additive from radish and spinach. It could be utilize radish and spinach as natural antimicrobial additive in preparation of chicken burger and to enhance the sensory properties.

Keywords: chemical composition; microbial loud; sensory characteristics, spinach, basil and radish; chicken burger.

\section{INTRODUCTION}

Poultry meats are characterized by their low fat and calorie, and it has highly susceptible lipids (Özünlü et al., 2018). Poultry proteins are subject to oxidation process causing some oxidation substances lead to degradation of quality of proteins, hence it results in some deterioration of texture, color, flavor and their nutritional characteristics (Estévez, 2011; Xiao, et al. 2011; Estévez, 2015). The replacement of red meat with chicken is an important to retard cholesterol levels, improve nutritive functional products and lowering fat meat so, it is a good as foodstuff processing into ready-to-eat meals (Barbut, 2002; Gross et al., 2002; Mohamed et al., 2014). Antioxidants are the important additive substances to prevent the oxidation of poultry meat and poultry meat products (Descalzo \& Sancho, 2008). Some synthetic antioxidants i.e. BHT and BHA are utilized to prevent off-flavor, rancidity and discoloration of poultry products, these synthetic substances cause some undesirable and toxic reactions, so several investigations were carried out to utilize natural antioxidants including phenolic, flavonoids and carotenoids from plant as substitute synthetic antioxidants (Shah, et al., 2014; Aziz and Karboune, 2017). Spinach has high content of bioactive compounds including phenolic acids, gallic, flavonoids, ferulic acids, carotenoids, lutein and carotene which represent antioxidant, antimicrobial and antioxidant and anti-carcinogenic substances (Fan, 2011 ; Bergman, et al., 2001; Bergman, et al., 2003 ; Bunea , 2008 ; Howard, and Pandjaitan, 2008; Vázquez et al., 2013 ). Many studies reported that basil has effectiveness as antimicrobial to inhibit gram-positive and gram-negative bacteria (Synowiec et al., 2014; Ngamakeue, et al., 2016; Singh, et al., 2018). Radish has high levels of phenolics, glucosinolates and many bioactive phytochemicals including antioxidant, antimicrobial, antidiabetic, phytochemicals and anthocyanins, which are owing to beneficial to human health (Oh and Rajashekar, 2009; Park et al., 2013; Zhang et al., 2019). This study was conducted to investigate some quality parameters including chemical composition, fatty acids and amino acids profile, sensory and microbiological evaluation for chicken burger prepared with addition of spinach, basil and radish to chicken burger formula as nontraditional alternatives during freeze storage at $\left(-20 \pm 2{ }^{\circ} \mathrm{C}\right)$.

\section{MATERIALS AND METHODS}

\section{Materials}

Chemicals were purchased from Sigma-Aldrich Company. All ingredients of chicken burger and chicken meat were obtained from local markets in Egypt.

\section{Methods}

\section{Preparation of chicken burger}

Fresh chicken burger was prepared according to formula (Table 1) as mentioned by Mohamed et al., (2014) prepared as follows ingredients in Table 1 with adding spinach, basil and radish (w/w) then chicken burger samples were packaged stored at $-20 \pm 2{ }^{\circ} \mathrm{C}$. Sensory, chemical and microbial evaluations of chicken burger samples under investigation were determined every two months during storage ( 8 months) at $-20 \pm 2{ }^{\circ} \mathrm{C}$. The treatments of chicken burger and their abbreviations showed in Table 2.

Table 1. Basal constituents of chicken burger formula

\begin{tabular}{lc}
\hline Ingredients & Gram \\
\hline Minced chicken meat & 875 \\
Fresh onion & 100 \\
Black pepper & 5 \\
All spice & 5 \\
Sodium chloride & 15 \\
\hline Total & 1000 \\
\hline
\end{tabular}

Table 2. Ingredients and abbreviations of chicken burger treatments

\begin{tabular}{lcc}
\hline Treatment & Ingredients & Abbreviation \\
\hline 1 & $\begin{array}{c}\text { Basal formula without any } \\
\text { additional ingredients } \\
\text { (Control) Table1 }\end{array}$ & Control burger ( CB) \\
& $\begin{array}{c}\text { Basal formula + spinach } \\
\text { 200gm }\end{array}$ & $\begin{array}{c}\text { Spinach burger (SB) } \\
2\end{array}$ \\
3 & Basal formula + basil 200 gm & basil burger (BB) \\
4 & $\begin{array}{c}\text { Basal formula }+ \text { radish 200gm } \\
\text { radish burger (RB) } \\
\text { Basal formula }+66.5\end{array}$ & Collected burger \\
5 & $\begin{array}{c}\text { spinach+66.5 basil +66.5 } \\
\text { radish (collected burger) }\end{array}$ & (Collected B) \\
\hline
\end{tabular}




\section{Chemical composition}

Gross chemical composition of chicken burger including moisture, lipid, protein, crude fiber and ash) determined according to official method (AOAC 2016), while total carbohydrates were calculated according to Egan et al., (1981) as the following:

Percent of total carbohydrates $=100-($ percent of (moisture + crude protein + total lipids + ash + crude fibers).

\section{Fatty acids profile}

Fatty acid profile was determined using gas chromatographic technique (GLC) as published by AOAC (2016).

\section{Amino acids composition}

Amino acid profile was determined according to AOAC (2016) by amino acid Analyzer technique.

\section{Sensory evaluation}

Sensory evaluation including appearance, color, texture, taste and odor,) of chicken burger samples were examined two months during storage ( 8 months) at $-20 \pm$ $2^{\circ} \mathrm{C}$ according to the method described by Mohamed et al., (2014).

\section{Microbial examination}

Total bacterial count was counted according to methods published by (APHA, 1992). Psychrophilic and spore-forming and bacteria counts determined according to FDA (2002). Total molds and yeasts were counted according to Oxoid (1998).

\section{Statistical analysis}

The statistical analysis was calculated according to method reported Bezerra et al. (2008)

\section{RESULTS AND DISCUSSION}

\section{Gross chemical composition of chicken burger samples}

Table (3) shows gross chemical composition of chicken burger treatments. The highest content of moisture found in $\mathrm{RB}$ treatment $(74.4 \%)$, while the lowest one was in $\mathrm{BB}$ treatment $(67.1 \%)$. The moisture content of other treatments ranged between $70.7 \%$ (CB) and $72.2 \%$ (collected B) and $73.2 \%$ (SB). Total lipids of the SB treatment were the lowest $(8.9 \%)$, while RB and collected B Treatments had the highest (11.1\%). Ash content ranged between $7.6 \%$ and $10.5 \%$, this is due to ingredients of chicken burger. Protein content of CB treatment was higher $(64.2 \%)$ while SB treatment was the lowest ratio of protein $(49.1 \%)$. Fiber content of BB treatment was the first $3.25 \%$ than the other treatments which ranged between $1.86 \%$ and 2.78 $\%$.Total carbohydrates of SB treatment were the highest $(31.65 \%)$. Meanwhile, the lowest observed in BB (14.20\%), and CB, collected B and RB treatments showed moderate content of carbohydrates $17.04 \%$, $19.02 \%$, and $23.03 \%$, respectively). These results are in consistent with those reported by Mohamed et al., (2014).
Table 3. Gross chemical composition of chicken burger samples (on dry weight basis)

\begin{tabular}{|c|c|c|c|c|c|}
\hline \multirow{2}{*}{$\begin{array}{l}\text { Constituents } \\
(\%)\end{array}$} & \multicolumn{5}{|c|}{ Treatments } \\
\hline & $\mathbf{C B}^{\mathbf{a}}$ & SB $^{\text {b }}$ & $\mathbf{B B}^{\mathbf{c}}$ & $\mathbf{R B}^{\mathrm{d}}$ & Collected Bf \\
\hline Moisture & 70.7 & 73.2 & 67.1 & 74.4 & 72.2 \\
\hline Total lipids & 9.2 & 8.9 & 9.02 & 11.1 & 11.1 \\
\hline Ash & 7.7 & 8.1 & 10.5 & 7.6 & 8.8 \\
\hline Crud protein & 64.2 & 49.1 & 63.03 & 56.1 & 58.3 \\
\hline Fiber & 1.86 & 2.25 & 3.25 & 2.17 & 2.78 \\
\hline $\begin{array}{l}\text { Total } \\
\text { carbohydrates }\end{array}$ & 17.04 & 31.65 & 14.20 & 23.03 & 19.02 \\
\hline
\end{tabular}

$\overline{C B}^{\mathrm{a}}$ : Basal formula without any additional ingredients (Control burger)

$\mathrm{SB}^{\mathrm{b}}$ : Basal formula + spinach $200 \mathrm{gm}$ (spinach burger)

$\mathrm{BB}^{\mathrm{c}}$ : Basal formula + basil $200 \mathrm{gm}$ (basil burger)

$\mathrm{RB}^{\mathrm{d}}$ : Basal formula+ radish $200 \mathrm{gm}$ ( radish burger)

Collected $\mathrm{B}^{\mathrm{f}}$ : Collected burger

Sensory evaluation

Table (4) shows sensory attributes including appearance, color, texture, taste and odor of chicken burger samples prepared with adding spinach, basil and radish during freeze storage period ( 8 months) at $-20 \pm 2{ }^{\circ} \mathrm{C}$. RD treatment was the highest followed by SB treatment, which rejected after 8 months while control sample, BB and Collected B treatments received significantly lower score for evaluated parameters and rejected after 6 months. Chicken burger samples prepared with radish and spinach were scored the best treatment compared to the other samples, this may be due to the effects of natural antimicrobial and antioxidants compounds of radish and spinach (Howard and Pandjaitan, 2008; Vázquez et al., 2013; Ngamakeue, et al., 2016; Singh, et al., 2018; ; Zhang et al., 2019).

Fatty acid profile

The data in Table (5) shows that total saturated and unsaturated fatty acids for oils extracted from chicken burger samples. The total saturated fatty acids for oils extracted from chicken burger samples recorded 29.65, 29.78 and $33.96 \%$, while the total unsaturated fatty acids amounted to $68.04,69.97$ and $65.77 \%$ for oils extracted from $\mathrm{CB}, \mathrm{SB}$ and $\mathrm{RB}$ treatments, respectively. The palmitic was predominant saturated fatty acids, while oleic acid was the first unsaturated fatty acids. These results confirmed with those of Mohamed et al., (2014). Chicken burger treatment (SB) contained higher level of unsaturated fatty acids. This is due to the addition of spinach that contains higher level of unsaturated fatty acids.

\section{Amino acid composition}

Data in Table (6) shows the essential and nonessential amino acids of chicken burger treatments. Lysine is the major essential amino acid and it ranged between $4.66 \%$ (SB sample) to $5.36 \%$ (control sample), followed by leucine with value ranged between $4.58 \%$ (SB sample) and $5.31 \%$ (control sample). Glutamic acid was the highest it recorded $10.12 \%$ for SB treatment and $12.19 \%$ for control sample. Aspartic acid was the second order of non-essential amino acids with the percentage ranged between 5.19 for SB treatment and $6.32 \%$ for control sample followed by, arginine and alanine These results agreement with obtained by Mikhail et al., (2014). 
Table 4. Changes in the sensory attributes of chicken burger during freeze storage $\left(-20 \pm 2^{\circ} \mathrm{C}\right)$

\begin{tabular}{|c|c|c|c|c|c|c|}
\hline \multirow{2}{*}{$\begin{array}{l}\text { Sensory } \\
\text { attributes }\end{array}$} & \multirow{2}{*}{$\begin{array}{l}\text { Storage } \\
\text { (Month) }\end{array}$} & \multicolumn{5}{|c|}{ Treatments } \\
\hline & & $\mathbf{C B}^{\mathrm{a}}$ & SB $^{\mathbf{b}}$ & $\mathbf{B B}^{\mathbf{c}}$ & $\mathbf{R B}^{\mathrm{d}}$ & Collected B ${ }^{f}$ \\
\hline \multirow{5}{*}{ Appearance } & Zero time & $8.1 \pm 2.3$ & $8.4 \pm 2.3$ & $6.8 \pm 1.5$ & $8.7 \pm 1.7$ & $6.7 \pm 0.98$ \\
\hline & 2 & $7.8 \pm 2.2$ & $7.8 \pm 1.7$ & $6.1 \pm 1.4$ & $8.3 \pm 1.4$ & $6 \pm 0.67$ \\
\hline & 4 & $7 \pm 1.9$ & $7.2 \pm 1.6$ & $4.7 \pm .78$ & $7.5 \pm 1.3$ & $5.1 \pm .66$ \\
\hline & 6 & (R) & $6.2 \pm 1.6$ & (R) & $6.3 \pm .99$ & ( \\
\hline & 8 & & ( & & (R) & \\
\hline \multirow{5}{*}{ Color } & Zero time & $8.2 \pm 2.2$ & $8.3 \pm 2.1$ & $7.1 \pm 1.4$ & $8.8 \pm 1.5$ & $7 \pm 0.93$ \\
\hline & 2 & $7.8 \pm 2.1$ & $7.8 \pm 1.9$ & $6.5 \pm 1.4$ & $8.1 \pm 1.4$ & $6 \pm 0.67$ \\
\hline & 4 & $6.7 \pm 1.8$ & $7.1 \pm 1.8$ & $5.1 \pm .90$ & $7.1 \pm 1.1$ & $5.1 \pm 0.66$ \\
\hline & 6 & (B) & $6.1 \pm 1.2$ & (B) & $6.1 \pm 0.79$ & (B) \\
\hline & 8 & & (R) & & (R) & \\
\hline \multirow{5}{*}{ Texture } & Zero time & $8.2 \pm 2.4$ & $8.4 \pm 2.3$ & $7.2 \pm 1.6$ & $8.5 \pm 1.5$ & $7.4 \pm 0.93$ \\
\hline & 2 & $7.9 \pm 2.1$ & $7.9 \pm 1.9$ & $6.1 \pm 1.3$ & $8 \pm 1.3$ & $6.3 \pm 1.1$ \\
\hline & 4 & $6.9 \pm 1.9$ & $7.2 \pm 1.7$ & $5.6 \pm 1.1$ & $7.2 \pm 1.3$ & $5.6 \pm 0.49$ \\
\hline & 6 & (R) & $6 \pm 1.4$ & (B) & $6.1 \pm 0.99$ & (B) \\
\hline & 8 & & ( & & ( & \\
\hline \multirow{5}{*}{ Taste } & Zero time & $8 \pm 2.1$ & $8 \pm 1.9$ & $7.1 \pm 1.5$ & $8.4 \pm 1.8$ & $7.3 \pm 0.90$ \\
\hline & 2 & $7.2 \pm 2.1$ & $7.5 \pm 1.7$ & $6 \pm 1.2$ & $7.7 \pm 1.3$ & $6.3 \pm 1.1$ \\
\hline & 4 & $6 \pm 1.7$ & $7.1 \pm 1.7$ & $4.1 \pm 0.60$ & $7.1 \pm 1.2$ & $3.9 \pm 0.73$ \\
\hline & 6 & (R) & $5.8 \pm 1.3$ & ( & $5.7 \pm 0.98$ & ( \\
\hline & 8 & & ( & & (R) & \\
\hline \multirow{5}{*}{ Oder } & Zero time & $8 \pm 2.3$ & $8.1 \pm 2.02$ & $7 \pm 1.4$ & $8.7 \pm 1.5$ & $7 \pm 0.93$ \\
\hline & 2 & $7.2 \pm 2.1$ & $7.4 \pm 1.7$ & $5.6 \pm 1.2$ & $7.7 \pm 1.4$ & $5.7 \pm 0.97$ \\
\hline & 4 & $5.8 \pm 1.6$ & $6.8 \pm 1.6$ & $3.5 \pm 0.49$ & $6.9 \pm 1.2$ & $4.1 \pm 0.93$ \\
\hline & 6 & ( & $5.2 \pm 1.2$ & (R) & $5.3 \pm 0.71$ & ( \\
\hline & 8 & & ( & & (R) & \\
\hline
\end{tabular}

®: At these points samples were rejected. Means \pm SD with the same letter in the same row are not significantly different $(\mathrm{P} \leq 0.05)$

$\mathrm{CB}^{\mathrm{a}}$ : Basal formula without any additional ingredients (Control burger)

$\mathrm{SB}^{\mathrm{b}}$ : Basal formula + spinach $200 \mathrm{gm}$. (spinach burger)

$\mathrm{BB}^{\mathrm{c}}$ : Basal formula+ basil $200 \mathrm{gm}$. (basil burger)

$\mathbf{R B}^{\mathrm{d}}$ : Basal formula+ radish $200 \mathrm{gm}$. (radish burger)

Collected $B^{f}$ : Collected burger

Table 5. Fatty acids profile of chicken burger samples

\begin{tabular}{|c|c|c|c|c|}
\hline \multirow{2}{*}{ Fatty acid (\%) } & & \multicolumn{3}{|c|}{ Treatments } \\
\hline & & \multirow{2}{*}{$\begin{array}{l}\mathbf{C B}^{\text {a }} \\
\text { N.D }\end{array}$} & \multirow{2}{*}{$\begin{array}{c}\mathbf{S B}^{\mathbf{b}} \\
\text { N.D }\end{array}$} & \multirow{2}{*}{$\begin{array}{l}\mathbf{R B}^{\mathbf{c}} \\
0.26\end{array}$} \\
\hline Capric acid & $(\mathrm{C} 10: 0)$ & & & \\
\hline Lauric acid & (C12:0) & N.D & N.D & 0.53 \\
\hline Myristic acid & $(\mathrm{C} 14: 0)$ & 0.59 & 0.60 & 1.82 \\
\hline Tetradecenoic acid & $(\mathrm{C} 14: 0 \omega 5)$ & N.D & N.D & 0.36 \\
\hline Pentadecanoic acid & (C15:0) & N.D & 0.15 & 0.60 \\
\hline Palmitic acid & $(\mathrm{C} 16: 0)$ & 21.81 & 21.91 & 22.81 \\
\hline Palmitioleic acid & $(\mathrm{C} 16: 1 \omega 7)$ & 4.42 & 4.38 & 3.69 \\
\hline Heptadecanoic acid & (C17:0) & 0.38 & 0.40 & 0.62 \\
\hline Decatrienoic acid & $(\mathrm{C} 16: 3 \omega 4)$ & N.D & N.D & 0.15 \\
\hline Stearic acid & (C18:0) & 6.87 & 6.72 & 6.85 \\
\hline Oleic acid & $(\mathrm{C} 18: 1 \omega 9)$ & 40.38 & 42.22 & 41.82 \\
\hline Linoleic acid & $(\mathrm{C} 18: 2 \omega 6)$ & 21.32 & 21.14 & 17.58 \\
\hline Decadienoic acid & $(\mathrm{C} 18: 2 \omega 4)$ & N.D & N.D & N.D \\
\hline Gamma linolenic acid & $(\mathrm{C} 18: 3 \omega 6)$ & 0.17 & 0.18 & 0.17 \\
\hline Linolenic acid & $(\mathrm{C} 18: 3 \omega 3)$ & 0.63 & 0.78 & 0.61 \\
\hline Octadecatetraenoic acid & $(\mathrm{C} 18: 4 \omega 3)$ & N.D & N.D & N.D \\
\hline Arachidic acid & (C20:0) & N.D & N.D & 0.11 \\
\hline 9 - Eicosaenoic acid & $(\mathrm{C} 20: 1 \omega 5)$ & 0.25 & 0.30 & N.D \\
\hline 11 - Eicosaenoic acid & $(\mathrm{C} 20: 1 \omega 5)$ & N.D & N.D & 0.25 \\
\hline Arachidonic acid & $(\mathrm{C} 20: 4 \omega 6)$ & 0.70 & 0.60 & 0.48 \\
\hline Erucic acid & $(\mathrm{C} 22: 1 \omega 9)$ & 0.17 & 0.14 & 0.15 \\
\hline Gadolic acid & $(\mathrm{C} 20: 1 \omega 9)$ & N.D & N.D & N.D \\
\hline Vaccinic acid & $(\mathrm{C} 18: 1 \omega 7)$ & N.D & N.D & 0.27 \\
\hline Docosenoic & $(\mathrm{C} 22: 1 \omega 11)$ & N.D & N.D & 0.13 \\
\hline Non identified fatty acid & & N.D & 0.23 & 0.21 \\
\hline Total saturated fatty acids & & 29.65 & 29.78 & 33.96 \\
\hline Total unsaturated fatty acids & & 68.04 & 69.97 & 65.77 \\
\hline Total fatty acids & & 97.69 & 99.75 & 99.73 \\
\hline $\begin{array}{l}\mathrm{CB}^{\mathrm{a}} \text { : } \\
\text { Basal formula withou } \\
\text { burger) }\end{array}$ & a to & ingr & ents & ontrol \\
\hline SB $^{\text {b }}$ : Basal formula + spinach & m. (spir &  & & \\
\hline $\mathrm{RB}^{\mathrm{c}}$ : Basal formula + radish 20 & 1. (radis & & & \\
\hline
\end{tabular}

Table 6. Amino acids composition of chicken burger samples

\begin{tabular}{|c|c|c|c|c|c|}
\hline \multirow{2}{*}{\multicolumn{3}{|c|}{$\begin{array}{l}\text { Amino acids } \\
(\%)\end{array}$}} & \multicolumn{3}{|c|}{ Treatments } \\
\hline & & & $\mathbf{C B}^{\mathbf{a}}$ & $\mathbf{S B}^{\mathbf{b}}$ & $\mathbf{R B}^{\mathbf{c}}$ \\
\hline \multirow{9}{*}{$\begin{array}{l}\text { Essential } \\
\text { amino acids }\end{array}$} & Therionine & (Thr) & 3.29 & 2.69 & 2.74 \\
\hline & Valine & (Val) & 3.67 & 2.78 & 3.02 \\
\hline & Methionine & (Met) & 1.75 & 1.56 & 1.64 \\
\hline & Isoleucine & (Ile) & 2.72 & 2.52 & 2.79 \\
\hline & Leucine & (Leu) & 5.31 & 4.58 & 4.80 \\
\hline & Tyrosine & (Tyr) & 1.85 & 1.78 & 1.36 \\
\hline & Phenyalanine & (Phe) & 2.74 & 2.42 & 2.52 \\
\hline & Lysine & (Lys) & 5.36 & 4.66 & 4.86 \\
\hline & Histidine & (His) & 1.54 & 1.54 & 1.60 \\
\hline \multicolumn{3}{|c|}{ Total essential amino acids } & 28.23 & 24.53 & 25.33 \\
\hline \multirow{8}{*}{$\begin{array}{l}\text { Non- } \\
\text { essential } \\
\text { amino acids }\end{array}$} & Aspartic & (Asp) & 6.32 & 5.19 & 5.52 \\
\hline & Serine & (Ser) & 2.91 & 2.50 & 2.41 \\
\hline & Glutamic & (Glu) & 12.19 & 10.12 & 10.25 \\
\hline & Prolin & (Pro) & 2.67 & 2.36 & 2.61 \\
\hline & Glycine & (Gly) & 4.14 & 3.54 & 3.62 \\
\hline & Alanine & (Ala) & 5.16 & 4.54 & 4.56 \\
\hline & Cystine & (Cys) & 0.68 & 0.57 & 0.81 \\
\hline & Arginine & (Arg) & 4.45 & 3.78 & 3.98 \\
\hline \multirow{2}{*}{\multicolumn{3}{|c|}{$\begin{array}{l}\text { Total non-essential amino acids } \\
\text { Total amino acid }\end{array}$}} & 38.52 & 32.6 & 33.76 \\
\hline & & & 66.75 & 57.13 & 59.09 \\
\hline
\end{tabular}

SB ${ }^{\text {b }}$ Basal formula + spinach $200 \mathrm{gm}$ (spinach burger)

$\mathbf{R B}^{\mathrm{c}}$ : Basal formula+ radish $200 \mathrm{gm}$. (radish burger)

\section{Microbial examination}

Total bacterial count, psychrophilic bacteria, spore forming bacteria, total molds and yeasts of chicken burger samples during freeze storage $\left(-20 \pm 2^{\circ} \mathrm{C}\right)$ were illustrated in Table (7). The best treatment to reduce the microbial load was $\mathrm{RB}$ followed by $\mathrm{SB}$ in chicken burger samples 
compared with other samples. These reductions in microbial load of $\mathrm{RB}$ and $\mathrm{SB}$ treatments due to the antimicrobial and antioxidant effect of spinach and radish. these results confirmed with those of Bergman, et al.,
(2003) ; Bunea , (2008) ; Howard, and Pandjaitan, (2008); Vázquez et al., (2013); Tenore et al., (2016); Ngamakeue, et al., (2016); Singh, et al., (2018).

Table7. Microbial load of chicken burger samples as affected by spinach, basil and radish during freeze storage ($20 \pm 2^{\circ} \mathrm{C}$ ).

\begin{tabular}{|c|c|c|c|c|c|c|}
\hline \multirow{2}{*}{$\begin{array}{l}\text { Microbiological } \\
\text { parameters }\end{array}$} & \multirow{2}{*}{$\begin{array}{l}\text { Storage } \\
\text { (Month) }\end{array}$} & \multicolumn{5}{|c|}{ Treatments } \\
\hline & & $\mathbf{C B}^{\mathbf{a}}$ & $\mathbf{S B}^{\mathbf{b}}$ & $\mathbf{B B}^{\mathbf{c}}$ & $\mathbf{R B}^{\mathbf{d}}$ & Collected B ${ }^{f}$ \\
\hline \multirow{5}{*}{ Total bacterial count } & Zero time & $5.3 \times 10^{2}$ & $5.1 \times 10^{2}$ & $5.3 \times 10^{2}$ & $5.1 \times 10^{2}$ & $5.4 \times 10^{2}$ \\
\hline & 2 & $7 \times 10^{2}$ & $6.4 \times 10^{2}$ & $6.7 \times 10^{2}$ & $6.3 \times 10^{2}$ & $6.5 \times 10^{2}$ \\
\hline & 4 & $9 \times 10^{2}$ & $8.2 \times 10^{2}$ & $8.9 \times 10^{2}$ & $7.9 \times 10^{2}$ & $7.7 \times 10^{2}$ \\
\hline & 6 & (R) & $9.6 \times 10^{2}$ & ( & $9.5 \times 10^{2}$ & ( \\
\hline & 8 & & (R) & & (R) & \\
\hline \multirow{5}{*}{ Psychrophilic bacteria } & Zero time & $2.6 \times 10^{2}$ & $2.5 \times 10^{2}$ & $2.6 \times 10^{2}$ & $2.5 \times 10^{2}$ & $2.6 \times 10^{2}$ \\
\hline & 2 & $3.5 \times 10^{2}$ & $3.3 \times 10^{2}$ & $3.4 \times 10^{2}$ & $3.2 \times 10^{2}$ & $3.6 \times 10^{2} 1$ \\
\hline & 4 & $5.8 \times 10^{2}$ & $5.2 \times 10^{2}$ & $5.3 \times 10^{2}$ & $5.4 \times 10^{2}$ & $5.5 \times 10^{2}$ \\
\hline & 6 & ( & $6.5 \times 10^{2}$ & (R) & $6.4 \times 10^{2}$ & (R) \\
\hline & 8 & & (R) & & ( & \\
\hline \multirow{5}{*}{ Spore-forming bacteria } & Zero time & $1.7 \times 10^{2}$ & $1.4 \times 10^{2}$ & $1.5 \times 10^{2}$ & $1.3 \times 10^{2}$ & $1.5 \times 10^{2}$ \\
\hline & 2 & $2.8 \times 10^{2}$ & $2.0 \times 10^{2}$ & $2.5 \times 10^{2}$ & $1.9 \times 10^{2}$ & $2.6 \times 10^{2}$ \\
\hline & 4 & $3.3 \times 10^{2}$ & $2.5 \times 10^{2}$ & $3.1 \times 10^{2}$ & $2.4 \times 10^{2}$ & $2.9 \times 10^{2}$ \\
\hline & 6 & ( & $3.1 \times 10^{2}$ & ( & $3.2 \times 10^{2}$ & ( \\
\hline & 8 & & ( & & ( & \\
\hline \multirow{5}{*}{ Total molds\& yeasts } & Zero time & $2.4 \times 10^{2}$ & $2.0 \times 10^{2}$ & $2.3 \times 10^{2}$ & $2.0 \times 10^{2}$ & $2.4 \times 10^{2}$ \\
\hline & 2 & $6.5 \times 10^{2}$ & $4.4 \times 10^{2}$ & $6.3 \times 10^{2}$ & $4.5 \times 10^{2}$ & $6.7 \times 10^{2}$ \\
\hline & 4 & $9 . .1 \times 10^{2}$ & $7.5 \times 10^{2}$ & $9.1 \times 10^{2}$ & $5.9 \times 10^{2}$ & $10.2 \times 10^{2}$ \\
\hline & 6 & ( & $8.8 \times 10^{2}$ & ( & $8.6 \times 10^{2}$ & ( \\
\hline & 8 & & (B) & & ( & \\
\hline
\end{tabular}

®: At these points samples were rejected

$\mathrm{CB}^{\mathrm{a}}$ : Basal formula without any additional ingredients (Control burger)

$\mathrm{SB}^{\mathrm{b}}$ : Basal formula + spinach $200 \mathrm{gm}$ (spinach burger)

$\mathrm{BB}^{\mathrm{c}}$ : Basal formula+ basil $200 \mathrm{gm}$ (basil burger)

$R^{d}$ : Basal formula+radish 200gm(radish burger)

Collected $B^{f}$ : Collected burger

\section{CONCLUSION}

This study aimed to utilize spinach, basil and radish in processing of chicken burger to improve their quality and lower cost of chicken burger. The results indicated that the some sensory evaluation including appearance, color, texture, taste and odor for $\mathrm{RB}$ treatment scoring was a significant $(\mathrm{P}>0.05)$ highly, followed by $\mathrm{SB}$ treatment during period's storage $\left(-20 \pm 2^{\circ} \mathrm{C}\right)$ which rejected after 8 months, will other treatments rejected after 6 months. Palmitic acid was the major saturated fatty acid, while oleic acid was the major unsaturated fatty acid. Lysine is the predominant essential amino acid and glutamic acid showed higher ratio of non-essential amino acids. A reduction in total molds and yeasts, total bacterial count, psychrophilic bacteria and spore-forming bacteria counted were observed in RB and SB treatments. The addition spinach and radish to burger formula improved quality attributes of chicken burger and prolong their shelf-life.

\section{REFERENCES}

AOAC (2016) Association of Official Analytical Chemist., EUA.

APHA (1992) Compendium of Methods for the Microbiological Examination of Foods"(2nd ed.), . American Puplic Heath Association, Washinton $D C$.
Aziz, B. S., T. R. Abdulwahid, A. M. Rasheed, G. O. Abdullah and M. H. Ahmed (2017) Polymer Blending as a Novel Approach for Tuning the SPR Peaks of Silver Nanoparticles. Polymers, 9.

Barbut, S. (2002) Poultry Products Processing. An Industry Guide. CRC Press LLC, Boca Raton, Florida.

Bergman, M., A. Perelman, Z. Dubinsky and S. Grossman (2003) Scavenging of reactive oxygen species by a novel glucurinated flavonoid antioxidant isolated and purified from spinach. Phytochemistry, 62, 753-762.

Bergman, M., L. Varshavsky, H. E. Gottlieb and S. Grossman (2001) The antioxidant activity of aqueous spinach extract: chemical identification of active fractions. Phytochemistry, 58, 143-152.

Bezerra, M. A., R. E. Santelli, E. P. Oliveira, L. S. Villar and L. A. Escaleira (2008) Response surface methodology (RSM) as a tool for optimization in analytical chemistry. Talanta, 76, 965-977.

Bunea, A., M. Andjelkovic, C. Socaciu, O. Bobis, M. Neacsu, R. Verhé and J. V. Camp (2008) Total and individual carotenoids and phenolic acids content in fresh, refrigerated and processed spinach (Spinacia oleracea L.). Food Chemistry, 108, 649-656.

Descalzo, A. M. and A. M. Sancho (2008) A review of natural antioxidants and their effects on oxidative status, odor and quality of fresh beef produced in Argentina. Meat Science, 79, 423-436. 
Egan, H. I., Kirk, R. S. and Sawyer, R. (1981 ) Person's. Chemical Analysis of Food. 8th ed., Churchill Livingstogne, Edintugh. .

Estévez, M. (2011) Protein carbonyls in meat systems: A review. Meat Science, 89, 259-279.

Estévez, M. (2015) Oxidative damage to poultry: From farm to fork. Poultry Science, 94, 1368-1378.

Fan, D., D. M. Hodges, J. Zhang, C. W. Kirby, X. Ji, S. J. Locke, A. T. Critchley and B. Prithiviraj (2011) Commercial extract of the brown seaweed Ascophyllum nodosum enhances phenolic antioxidant content of spinach (Spinacia oleracea L.) which protects Caenorhabditis elegans against oxidative and thermal stress. Food Chemistry, 124, 195-202.

FDA, (2002). Bacteriological Analytical Manual. 9th Ed., AOAC Int., Arlington, VA,USA.

Gross, J. L., T. Zelmanovitz, C. C. Moulin, V. De Mello, M. Perassolo, C. Leitão, A. Hoefel, A. Paggi and M. J. Azevedo (2002) Effect of a Chicken-Based Diet on Renal Function and Lipid Profile in Patients With Type 2 Diabetes. Diabetes Care, 25, 645.

Howard, L. and N. Pandjaitan (2008) Pressurized liquid extraction of flavonoids from spinach. J. Food Science 73, 151-157.

Mikhail, W. Z. A., H. M. Sobhy, M. F. Khallaf, H. M. Z. Ali, S. A. El-askalany and M. M. Ezz El-Din (2014) Suggested treatments for processing high nutritive value chicken burger. Annals of Agricultural Sciences, 59, 41-45.

Mohamed, F. K., H. M. Sobhy, W. Z. Azer, M. E. E.-D. Manal, H. M. Z. Ali and S. A. El-askalany (2014) Fatty acid profile, antioxidant activity of various suggested chicken burger treatments. Annals of Agricultural Sciences, 59, 47-51.

Ngamakeue, N. and P. Chitprasert (2016) Encapsulation of Holy Basil Essential Oil in Gelatin: Effects of Palmitic Acid in Carboxymethyl Cellulose Emulsion Coating on Antioxidant and Antimicrobial Activities. Food and Bioprocess Technology, 9, 1735-1745.

Oh, M. M. and C. B. Rajashekar (2009) Antioxidant content of edible sprouts: effects of environmental shocks. J. Sci. Food Agric. 89, , 89, 2221-2227.
Oxoid, T. o. M. (1998) (8th ed.)Oxid ltd., Wade Road, Basingstoke, Hampshire, R G24 8PW, Engand.

Özünlü, O., H. Ergezer and R. Gökçe (2018) Improving physicochemical, antioxidative and sensory quality of raw chicken meat by using acorn extracts. $L W T$, 98, 477-484.

Park, W. T., Y. B. Kim, J. M. Seo, S.-J. Kim, E. Chung, J.H. Lee and S. U. Park (2013) Accumulation of Anthocyanin and Associated Gene Expression in Radish Sprouts Exposed to Light and Methyl Jasmonate. Journal of Agricultural and Food Chemistry, 61, 4127-4132.

Shah, M. A., S. J. D. Bosco and S. A. Mir (2014) Plant extracts as natural antioxidants in meat and meat products. Meat Science, 98, 21-33.

Singh, S., K. K. Gaikwad and Y. S. Lee (2018) Antimicrobial and antioxidant properties of polyvinyl alcohol bio composite films containing seaweed extracted cellulose nano-crystal and basil leaves extract. International Journal of Biological Macromolecules, 107, 1879-1887.

Synowiec, A., M. Gniewosz, K. Kraśniewska, J. L. Przybył, K. Bączek and Z. Węglarz (2014) Antimicrobial and antioxidant properties of pullulan film containing sweet basil extract and an evaluation of coating effectiveness in the prolongation of the shelf life of apples stored in refrigeration conditions. Innovative Food Science \& Emerging Technologies, 23, 171-181.

Vázquez, E., M. R. García-Risco, L. Jaime, G. Reglero and T. Fornari (2013) Simultaneous extraction of rosemary and spinach leaves and its effect on the antioxidant activity of products. The Journal of Supercritical Fluids, 82, 138-145.

Xiao, S., W. G. Zhang, E. J. Lee, C. W. Ma and D. U. Ahn (2011) Effects of diet, packaging, and irradiation on protein oxidation, lipid oxidation, and color of raw broiler thigh meat during refrigerated storage. . Poultry Science,, 90, 1348-1357.

Zhang, X., J. Wei, J. Tian, N. Li, L. Jia, W. Shen and J. Cui (2019) Enhanced anthocyanin accumulation of immature radish microgreens by hydrogen-rich water under short wavelength light. Scientia Horticulturae, 247, 75-85.

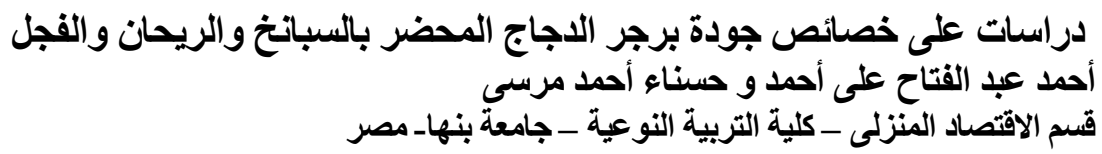

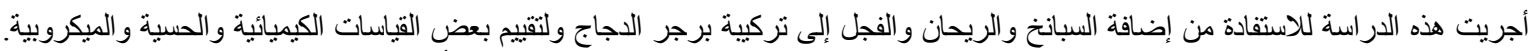



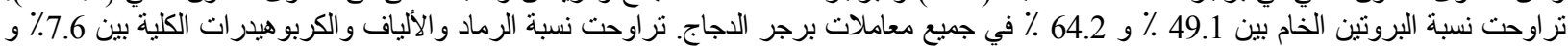

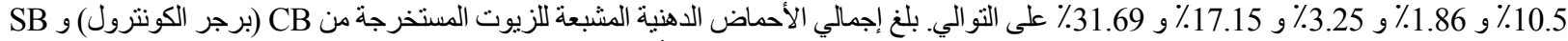

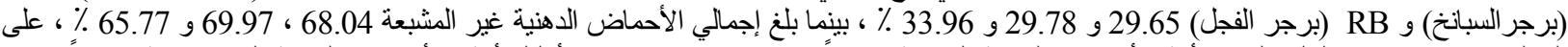

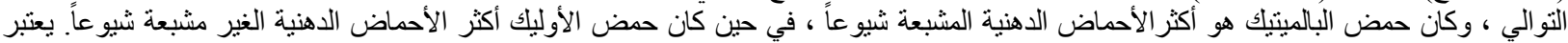

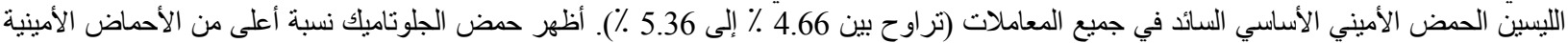



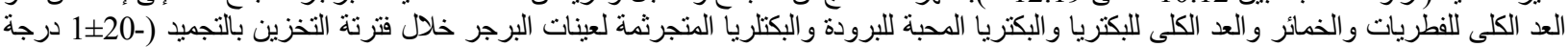

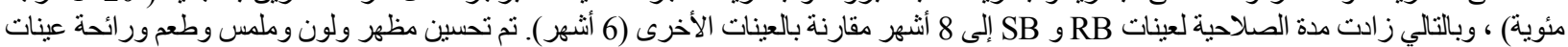

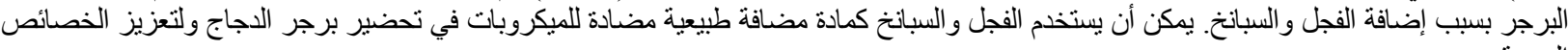

